\title{
COMPOSITION OPERATORS BETWEEN ALGEBRAS OF DIFFERENTIABLE FUNCTIONS
}

\author{
JOAQUÍN M. GUTIÉRREZ AND JOSÉ G. LLAVONA
}

\begin{abstract}
Let $E, F$ be real Banach spaces, $U \subseteq E$ and $V \subseteq F$ nonvoid open subsets and $C^{k}(U)$ the algebra of real-valued $k$-times continuously Fréchet differentiable functions on $U$, endowed with the compact open topology of order $k$. It is proved that, for $m \geq p$, the nonzero continuous algebra homomorphisms $A: C^{m}(U) \rightarrow C^{p}(V)$ are exactly those induced by the mappings $g: V \rightarrow U$ satisfying $\phi \circ g \in C^{p}(V)$ for each $\phi \in E^{*}$, in the sense that $A(f)=f \circ g$ for every $f \in C^{m}(U)$. Other homomorphisms are described too. It is proved that a mapping $g: V \rightarrow E^{* *}$ belongs to $C^{k}\left(V,\left(E^{* *}, w^{*}\right)\right)$ if and only if $\phi \circ g \in C^{k}(V)$ for each $\phi \in E^{*}$. It is also shown that if a mapping $g: V \rightarrow E$ verifies $\phi \circ g \in C^{k}(V)$ for each $\phi \in E^{*}$, then $g \in C^{k-1}(V, E)$.
\end{abstract}

\section{INTRODUCTION}

Homomorphisms between function algebras on Banach spaces have been described in recent years by various authors: for instance, $[3,13]$ deal with algebras of continuous functions, and $[2,11]$ with algebras of differentiable functions.

Let $C_{w u}^{k}(E)$ denote the algebra of $k$-times continuously differentiable realvalued functions on the real Banach space $E$ such that the functions and their derivatives are weakly uniformly continuous on bounded subsets. It is proved in [2] that the nonzero (algebra) homomorphisms $A: C_{w u}^{m}(E) \rightarrow C_{w u}^{p}(F) \quad(m \geq$ $p$ ) are exactly those induced by the mappings $g: F \rightarrow E^{* *}$ satisfying $\phi \circ$ $g \in C_{w u}^{p}(F)$ for each $\phi \in E^{*}$, in the sense that $A(f)=\tilde{f} \circ g$ where $\tilde{f}$ is the extension of $f \in C_{w u}^{m}(E)$ to the bidual of $E$. Since $C_{w u}^{m}(E)$ is a real Fréchet algebra, it can be proved that these homomorphisms are automatically continuous.

It was then natural to try to describe the continuous homomorphisms $A$ : $C^{m}(U) \rightarrow C^{p}(V) \quad(m \geq p)$, where $U \subseteq E$ and $V \subseteq F$ are open, and $C^{m}(U)$ is the algebra of all $m$-times continuously differentiable real-valued functions on $U$ endowed with one of the natural topologies: the compact open topology of order $m\left(\tau_{u}^{m}\right)$ and the compact-compact topology of order $m\left(\tau_{c}^{m}\right)$ (definitions below). This is the problem we solve here. Some of our results are the following:

(a) $A$ is $\tau_{u}^{m}-\tau_{u}^{p}$ continuous if and only if it is induced by a mapping $g: V \rightarrow U$ satisfying $\phi \circ g \in C^{p}(V) \quad\left(\phi \in E^{*}\right)$;

Received by the editors December 4, 1990 and, in revised form, May 9, 1991.

1991 Mathematics Subject Classification. Primary 46E25, 46G05; Secondary 26E15.

Key words and phrases. Differentiable mappings between Banach spaces, algebras of differentiable functions, homomorphisms, composition operators.

Research supported in part by DGICYT Project PB 87-1031 (Spain). 
(b) $A$ is $\tau_{c}^{m}-\tau_{u}^{p}$ continuous if and only if it is induced by a $C^{p}$ mapping $g: V \rightarrow U$ whose derivatives are compact polynomials.

These results are proved by first showing that if $g: V \rightarrow E$ satisfies $\phi$ 。 $g \in C^{p}(V)\left(\phi \in E^{*}\right)$, then $g \in C^{p}\left(V, E_{w^{*}}^{* *}\right)$ where $E_{w^{*}}^{* *}$ is the bidual of $E$ endowed with the weak-star topology. As a consequence we prove that such a function belongs to $C^{p-1}(V, E)$. This is the real analogue of a well-known result on holomorphic mappings: if $E$ and $F$ are complex Banach spaces, and $V \subseteq F$ is a nonvoid open subset, then a mapping $g: V \rightarrow E$ is holomorphic if and only if, for every $\phi \in E^{*}, \phi \circ g$ is holomorphic [17, 8.12]. In the differentiable case, this property is stated in [7, 2.6.2] but we do not know of any published proof.

\section{NOTATIONS, DEFINITIONS, AND PRELIMINARY RESULTS}

Throughout, $E, F$, and $G$ will be real Banach spaces, $E^{*}$ and $E^{* *}$, the dual and the bidual, respectively, of $E$. The weak-star topology is denoted by $w^{*}$ and we write $E_{w^{*}}^{*}=\left(E^{*}, w^{*}\right) . B_{E}$ is the closed unit ball of $E$ and $\mathscr{O}(E)$ the family of all nonempty open subsets of $E$. Given a set $A \subset E, A^{\circ}$ stands for its polar. $E_{c}^{*}$ will denote the space $E^{*}$ endowed with the topology of compact convergence. $\mathbb{R}$ represents the real line, $\mathbb{N}$ the set of natural numbers including 0 , and $\mathbb{N}^{*}=\mathbb{N} \cup\{\infty\}$.

For $n \in \mathbb{N}, L\left({ }^{n} E, F\right)$ denotes the Banach space of all $n$-linear continuous mappings from $E^{n}=E \times \stackrel{(n)}{\cdots} \times E$ into $F$, endowed with the norm

$$
\|A\|=\sup \left\{\left\|A\left(x_{1}, \ldots, x_{n}\right)\right\|:\left\|x_{i}\right\| \leq 1,1 \leq i \leq n\right\} \text {. }
$$

$L(E, F)$ stands for $L\left({ }^{1} E, F\right) . L_{s}\left({ }^{n} E, F\right)$ is the subspace of $L\left({ }^{n} E, F\right)$ consisting of all symmetric $n$-linear continuous mappings. $\mathscr{P}\left({ }^{n} E, F\right)$ will denote the Banach space of all $n$-homogeneous continuous polynomials from $E$ to $F$, endowed with the norm $\|P\|=\sup \{\|P(x)\|:\|x\| \leq 1\}$.

The map $T: L_{s}\left({ }^{n} E, F\right) \rightarrow \mathscr{P}\left({ }^{n} E, F\right)$, given by $T(A)=P$ with $P(x)=$ $A(x, \stackrel{(n)}{.}, x)$, is a topological isomorphism [18, §3]. We write $A=\widehat{P}$ and say that $\widehat{P}$ is the symmetric $n$-linear continuous mapping associated to the polynomial $P$. The following inequalities hold:

$$
\|P\| \leq\|\widehat{P}\| \leq\left(n^{n} / n !\right)\|P\| \text {. }
$$

$\mathscr{P}\left({ }^{n} E, F\right)_{c}$ will be the space of $n$-homogeneous continuous polynomials endowed with the topology of uniform convergence on compact subsets of $E$. $\mathscr{P}_{K}\left({ }^{n} E, F\right)$, the space of $n$-homogeneous compact polynomials from $E$ to $F$, consists of all $P \in \mathscr{P}\left({ }^{n} E, F\right)$ such that $P\left(B_{E}\right)$ is relatively compact in $F . \mathscr{P}_{w b}\left({ }^{n} E, F\right)$ is the set of all $P \in \mathscr{P}\left({ }^{n} E, F\right)$ whose restrictions to bounded subsets of $E$ are weakly (uniformly) continuous; it is a subspace of $\mathscr{P}_{K}\left({ }^{n} E, F\right)$ $[16,4.5 .9$ and 4.1.1].

If $U \in \mathscr{O}(E)$, then $C^{m}(U, F)$ (resp. $C_{H}^{m}(U, F)$ ) denotes the vector space of all $m$-times continuously differentiable mappings in the Fréchet (resp. Hadamard) sense from $U$ to $F$. General results on differentiable mappings may be seen in [14] or [22].

For $U \in \mathscr{O}(E)$, we define two topologies in the space $C^{m}(U, F)$ that only coincide when $E$ is finite dimensional:

(a) the topology $\tau_{u}^{m}$ (compact open of order $m$ ), given by the seminorms 
$p_{K}(f)=\sup \left\{\left\|d^{j} f(x)\right\|: x \in K, 0 \leq j \leq m\right\}$ (where $d^{0} f=f$ ), for each compact subset $K \subset U$.

(b) the topology $\tau_{c}^{m}$ (compact-compact of order $m$ ), introduced by Llavona [15] and Prolla [19], given by the seminorms

$$
p_{K, L}(f)=\sup \left\{\|f(x)\|,\left\|d^{j} f(x)(y)\right\|: x \in K, \quad y \in L, \quad 1 \leq j \leq m\right\}
$$

for every pair of compact subsets $K \subset U$ and $L \subset E$.

The space $C_{H}^{m}(U, F)$ is always endowed with the topology $\tau_{c}^{m}$.

In $[1,4]$ the following classes of differentiable functions were introduced:

(a) the space $C_{K}^{m}(U, F)$ of compact differentiable mappings of order $m$, consisting of all $f \in C^{m}(U, F)$ such that for every $x \in U, d^{j} f(x) \in$ $\mathscr{P}_{K}\left({ }^{j} E, F\right) \quad(1 \leq j \leq m)$.

(b) $C_{c}^{m}(U, F)$ is the space of all mappings $f \in C^{m}(U, F)$ such that for each $x \in U$ and $j \in \mathbb{N},(1 \leq j \leq m), d^{j} f(x) \in \mathscr{P}_{w b}\left({ }^{j} E, F\right)$;

(c) $C_{w u}^{m}(U, F)$ consists of all $f \in C_{c}^{m}(U, F)$ such that the derivative map $d^{j} f: U \rightarrow \mathscr{P}_{w b}\left({ }^{j} E, F\right)$ is weakly uniformly continuous on bounded subsets of $U$, for $0 \leq j \leq m$.

If a mapping between Banach spaces is weakly uniformly continuous on bounded subsets, then it takes bounded sets into precompact ones [16, 4.1.1]. Therefore, it is possible to endow the space $C_{w u}^{m}(U, F)$ with the topology $\tau_{b}^{m}$ generated by the family of seminorms

$$
p_{B}(f)=\sup \left\{\left\|d^{j} f(x)\right\|: x \in B, 0 \leq j \leq m\right\},
$$

for each bounded set $B \subset U$.

If $E^{*}$ has the bounded approximation property, then the polynomials of finite type from $E$ to $F$ are dense in $\left(C_{c}^{m}(E, F), \tau_{u}^{m}\right)$ and in $\left(C_{w u}^{m}(E, F), \tau_{b}^{m}\right)$ [4].

Throughout, when the range space is omitted, it is understood to be $\mathbb{R}$. Thus, $C^{m}(U)=C^{m}(U, \mathbb{R}), \mathscr{P}\left({ }^{n} E\right)=\mathscr{P}\left({ }^{n} E, \mathbb{R}\right)$, etc.

\section{COMPOSITION OPERATORS}

In this section, it is shown that every nonzero continuous homomorphism between algebras of differentiable functions is induced by a mapping. By standard techniques (see for instance [10]), the following result may be proved:

2.1. Proposition. Let $p \in \mathbb{N}^{*} \backslash\{0\}$. If $\Phi:\left(C^{p}(U), \tau_{u}^{p}\right) \rightarrow \mathbb{R}$ is a nonzero continuous homomorphism, then there is a unique $x \in U$ such that $\Phi(f)=f(x)$ for every $f \in C^{p}(U)$.

This result remains valid when replacing the algebra $\left(C^{p}(U), \tau_{u}^{p}\right)$ by $\left(C^{p}(U)\right.$, $\left.\tau_{c}^{p}\right)$ or $C_{H}^{p}(U)$.

2.2 Theorem. Let $p, m \in \mathbb{N}^{*} \backslash\{0\}, U \in \mathscr{O}(E), V \in \mathscr{O}(F)$ and let a nonzero algebra homomorphism $A: C^{m}(U) \rightarrow C^{p}(V)$ be given. $A$ is supposed to be continuous when $C^{m}(U)$ is endowed with one of the topologies $\tau_{u}^{m}, \tau_{c}^{m}$ and $C^{p}(V)$ is also endowed with one of the topologies $\tau_{u}^{p}, \tau_{c}^{p}$. Then there is a unique map $g: V \rightarrow U$ such that $A(f)=f \circ g$ for each $f \in C^{m}(U)$.

Proof. Let $\delta_{y}$ be the evaluation map at a point $y \in V$. Then $\delta_{y} \circ A$ is a nonzero continuous homomorphism from $C^{m}(U)$ to $\mathbb{R}$, so there is a unique 
$x \in U$ such that $A(f)(y)=\delta_{y} \circ A(f)=f(x)$ for every $f \in C^{m}(U)$. If $g: V \rightarrow U$ is the mapping taking $y$ into $x$, then $A(f)=f \circ g$ for each $f \in C^{m}(U)$. It is easy to see that $g$ must be unique.

Since $E^{*} \subset C^{m}(U)$, we have $\phi \circ g=A(\phi) \in C^{p}(V)$ for each $\phi \in E^{*}$.

The theorem still holds for algebras of Hadamard differentiable functions.

\section{WEAKLY DIFFERENTIABLE MAPPINGS}

For $V \in \mathscr{O}(F)$, suppose the map $g: V \rightarrow E$ is such that $\phi \circ g \in C^{p}(V)$ for each $\phi \in E^{*}$. We shall then say that $g$ is weakly $C^{p}$, or weakly $p$-times continuously differentiable, and prove that $g \in C^{p}\left(V, E_{w^{*}}^{* *}\right)$. This result will be important in the following section.

As a consequence, it is also proved that every mapping $g$ as above belongs to the space $C^{p-1}(V, E)$. This property was stated in [7, 2.6.2] but we have not found any proof of this fact in the literature, unless $F$ is finite dimensional: see the appendix of [20] for $F=\mathbb{R}^{n}$.

We first need a lemma on polynomial spaces:

3.1. Lemma. For $j \in \mathbb{N} \backslash\{0\}, \mathscr{P}\left({ }^{j} F, E^{* *}\right)$ and $\mathscr{P}\left({ }^{j} F, E_{w^{*}}^{* *}\right)$ coincide as vector spaces.

Proof. It is clear that $\mathscr{P}\left({ }^{j} F, E^{* *}\right) \subset \mathscr{P}\left({ }^{j} F, E_{w^{*}}^{* *}\right)$. Let now a polynomial $P \in$ $\mathscr{P}\left({ }^{j} F, E_{w^{*}}^{* *}\right)$ be given. Then $P\left(B_{F}\right)$ is weak-star bounded (so norm bounded) in $E^{* *}$ and hence $P \in \mathscr{P}\left({ }^{j} F, E^{* *}\right)[17,2.4]$.

3.2. Theorem. Let $g: V \rightarrow E^{* *}$ be a mapping, where $V \in \mathscr{O}(F)$. Let $p \in \mathbb{N}^{*}$. Then $g \in C^{p}\left(V, E_{w^{*}}^{* *}\right)$ if and only if $\phi \circ g \in C^{p}(V)$, for every $\phi \in E^{*}$.

Proof. Take first $p=0$. If $\phi \circ g$ is continuous for each $\phi \in E^{*}$, then given $x \in V, \varepsilon>0, \phi \in E^{*}$, there is $\delta>0$ so that whenever $\|x-y\|<\delta \quad(y \in V)$, we have $|\phi(g(x))-\phi(g(y))|<\varepsilon$, and thus $g: V \rightarrow E_{w^{*}}^{* *}$ is continuous. The converse is obvious.

Let now $p \geq 1$. For the nontrivial part, suppose $\phi \circ g \in C^{p}(V) \quad(\phi \in$ $\left.E^{*}\right)$. Following [2, Theorem 3.3], define the map $g_{j}: V \rightarrow \mathscr{P}\left({ }^{j} F, E_{w^{*}}^{* *}\right)$ by $g_{j}(y)(x)(\phi)=d^{j}(\phi \circ g)(y)(x)$, for $y \in V, x \in F, \phi \in E^{*}$, and $1 \leq j \leq p$.

The maps $g_{j}$ are well defined. Indeed, for every $\phi \in E^{*}, y \in V, x \in F$,

$$
d(\phi \circ g)(y)(x)=\lim _{t \rightarrow 0} \frac{1}{t}((\phi \circ g)(y+t x)-(\phi \circ g)(y)) .
$$

There is $\delta>0$ such that the set $\left\{t^{-1}(g(y+t x)-g(y)): 0<|t| \leq \delta\right\}$ is bounded: otherwise we would find a sequence $t_{n} \rightarrow 0$ in $\mathbb{R}$ so that the vectors

$$
\zeta_{n}=\left(1 / t_{n}\right)\left(g\left(y+t_{n} x\right)-g(y)\right)
$$

verify $\left\|\zeta_{n}\right\| \geq n$, and then $\lim _{n} \zeta_{n}(\phi)$ would not exist for some $\phi \in E^{*}$, in contradiction with (1). Hence, there is $M>0$ such that

$$
\sup \left\{\left|g_{1}(y)(x)(\phi)\right|: \phi \in B_{E^{*}}\right\}=\sup \left\{|d(\phi \circ g)(y)(x)|: \phi \in B_{E^{*}}\right\} \leq M,
$$

so $g_{1}(y)(x) \in E^{* *}$. It is now easily proved that $g_{1}(y) \in L\left(F, E_{u w^{*}}^{* *}\right)$.

By induction and using similar arguments, it is not difficult to see that $g_{j}(y)(x)$ $\in E^{* *}$ and $g_{j}(y) \in \mathscr{P}\left({ }^{j} F, E_{w^{*}}^{* *}\right) \quad(1 \leq j \leq p)$. 
We shall now prove that $g_{j}=d^{j} g$. For each bounded set $B \subset F, y \in V$, and $\phi \in E^{*}$ :

$$
\begin{aligned}
\lim _{t \rightarrow 0} t^{-1}\left(g(y+t x)-g(y)-g_{1}(y)(t x)\right)(\phi) \\
\quad=\lim _{t \rightarrow 0} t^{-1}((\phi \circ g)(y+t x)-(\phi \circ g)(y)-d(\phi \circ g)(y)(t x))=0,
\end{aligned}
$$

uniformly for $x \in B$. Hence,

$$
\lim _{t \rightarrow 0} t^{-1}\left(g(y+t x)-g(y)-g_{1}(y)(t x)\right)=0
$$

in $E_{w^{*}}^{* *}$ uniformly for $x \in B$, and $g_{1}$ is the derivative of $g$.

Assume now that $g_{1}, \ldots, g_{j-1}$ are the first $j-1$ derivatives of $g$, with $j \leq p$. For $y \in V$ fixed, let $u_{j}^{y}: F \rightarrow \mathscr{P}\left({ }^{j-1} F, E_{w^{*}}^{* *}\right)$ be the linear mapping given by

$$
u_{j}^{y}(x)(z)=\hat{g}_{j}(y)(x, z,(j-1), z) \quad(x, z \in F),
$$

where $\hat{g}_{j}(y)$ is the symmetric $j$-linear map associated to the polynomial $g_{j}(y)$. We only have to show that, for any bounded set $B \subset F$, one has

$$
\lim _{t \rightarrow 0} t^{-1}\left(g_{j-1}(y+t x)-g_{j-1}(y)-u_{j}^{y}(t x)\right)=0
$$

in $\mathscr{P}\left({ }^{j-1} F, E_{w^{*}}^{* *}\right)$, uniformly for $x \in B$.

Fix $\phi \in E^{*}$. Let $v_{j, \phi}^{y}$ be the linear mapping associated to $d^{j}(\phi \circ g)(y)$, as we did above for $u_{j}^{y}$. Since $\phi \circ g \in C^{j}(V)$, we have

$$
\lim _{t \rightarrow 0} t^{-1}\left(d^{j-1}(\phi \circ g)(y+t x)(z)-d^{j-1}(\phi \circ g)(y)(z)-v_{j, \phi}^{y}(t x)(z)\right)=0
$$

uniformly for $x, z \in B$. Now, $v_{j, \phi}^{y}(t x)(z)=u_{j}^{y}(t x)(z)(\phi)$, so we have

$$
\lim _{t \rightarrow 0} t^{-1}\left(g_{j-1}(y+t x)(z)-g_{j-1}(y)(z)-u_{j}^{y}(t x)(z)\right)(\phi)=0
$$

uniformly for $x, z \in B$, and (2) follows.

Finally, $g_{j}$ is continuous. Indeed, let $B \subset F$ be bounded, $x \in V$ fixed, $\varepsilon>0, \phi \in E^{*}$. By the continuity of $d^{j}(\phi \circ g): V \rightarrow \mathscr{P}\left({ }^{j} F\right)$, there is a $\delta>0$ such that whenever $\|x-y\|<\delta \quad(y \in V)$, we have $\left|g_{j}(x)(z)(\phi)-g_{j}(y)(z)(\phi)\right|=\left|d^{j}(\phi \circ g)(x)(z)-d^{j}(\phi \circ g)(y)(z)\right|<\varepsilon \quad(z \in B)$. Hence, $g_{j}$ is continuous and therefore $g \in C^{j}\left(V, E_{w^{*}}^{* *}\right)$.

3.3. Lemma. Let $p \in \mathbb{N}, V \in \mathscr{O}(F)$, and $g \in C^{p}\left(V, E_{w^{*}}^{* *}\right)$. For $j \in$ $\{1, \ldots, p\}, K \subset V$ compact, and $B \subset F$ bounded, the set $d^{j} g(K)(B)$ is bounded in $E^{* *}$.

Proof. By the continuity of $d^{j} g, d^{j} g(K)$ is compact in $\mathscr{P}\left({ }^{j} F, E_{w^{*}}^{* *}\right)$. Since, for every $\phi \in E^{*}$, the set

$$
V(\phi, B, 1)=\left\{P \in \mathscr{P}\left({ }^{j} F, E_{w^{*}}^{* *}\right):|P(y)(\phi)|<1(y \in B)\right\}
$$

is a zero neighborhood, we can find $x_{1}, x_{2}, \ldots, x_{n} \in K$ such that

$$
d^{j} g(K) \subset \bigcup_{i=1}^{n}\left(d^{j} g\left(x_{i}\right)+V(\phi, B, 1)\right) \text {. }
$$

Since $d^{j} g\left(x_{i}\right)(B)$ is bounded, we conclude that $d^{j} g(K)(B)$ is bounded.

The segment whose bounds are $x, y \in F$ will be denoted by $[x, y]$. 
3.4. Proposition. Let $p \in \mathbb{N}^{*} \backslash\{0\}$ and $V \in \mathscr{O}(F)$. The space $C^{p}\left(V, E_{w^{*}}^{* *}\right)$ is contained in $C^{p-1}\left(V, E^{* *}\right)$.

Proof. Without loss of generality, we can suppose $V$ convex.

For $p=1$, let $K \subset V$ be a convex compact set, $x \in K$ fixed. For $y \in K$ and $g \in C^{1}\left(V, E_{w^{*}}^{* *}\right)$, we have, by the mean value theorem [22, 1.3.3]:

$$
\begin{aligned}
\|g(x)-g(y)\| & =\sup \left\{|\phi(g(x)-g(y))|: \phi \in B_{E^{*}}\right\} \\
& \leq\|x-y\| \sup \left\{\|\phi \circ d g(z)\|: z \in[x, y], \phi \in B_{E^{*}}\right\} \\
& \leq\|x-y\| \cdot M
\end{aligned}
$$

where $M>0$ is given by Lemma 3.3. Hence $g: V \rightarrow E^{* *}$ is continuous.

For $p \geq 2$, take $g \in C^{p}\left(V, E_{w^{*}}^{* *}\right), y \in V$ fixed, $B \subset F$ bounded, $h \in B$, $\phi \in E^{*}$, and $2 \leq j \leq p$.

Let $u_{j}^{y}: F \rightarrow \mathscr{P}\left({ }^{j-1} F, E_{w^{*}}^{* *}\right)$ be the continuous linear map given by

$$
u_{j}^{y}(x)(z)=\hat{d}^{j} g(y)(x, z, \stackrel{(j-1)}{\cdots}, z) \quad(x, z \in F) .
$$

Consider the differentiable map $f: \mathbb{R} \rightarrow \mathscr{P}\left({ }^{j-2} F\right)$ given by

$$
f(t)=\phi \circ\left(d^{j-2} g(y+t h)-u_{j-1}^{y}(t h)\right) .
$$

By the mean value theorem:

$$
\frac{1}{|t|}\|f(t)-f(0)\| \leq \sup \left\{\left\|f^{\prime}(\lambda)\right\|: \lambda \in[0, t]\right\}
$$

Now,

$$
\begin{aligned}
\left\|f^{\prime}(\lambda)\right\| & =\left\|\phi \circ\left(u_{j-1}^{y+\lambda h}(h)-u_{j-1}^{y}(h)\right)\right\| \\
& =\sup _{z \in B_{F}}\left\{\left|\phi \circ\left(\hat{d}^{j-1} g(y+\lambda h)-\hat{d}^{j-1} g(y)\right)(h, z, \stackrel{(j-2)}{\cdots}, z)\right|\right\} \\
& \leq C\left\|d^{j-1}(\phi \circ g)(y+\lambda h)-d^{j-1}(\phi \circ g)(y)\right\|\|h\|,
\end{aligned}
$$

where $C=(j-1)^{j-1} /(j-1)$ ! (see $\left.\S 1\right)$.

Applying again the mean value theorem, we have

$$
\left\|d^{j-1}(\phi \circ g)(y+\lambda h)-d^{j-1}(\phi \circ g)(y)\right\| \leq\|\lambda h\| \sup \left\{\left\|\phi \circ u_{j}^{y+\theta \lambda h}\right\|: \theta \in[0,1]\right\} \text {. }
$$

Moreover,

$$
\left\|\phi \circ u_{j}^{y+\theta \lambda h}\right\| \leq\left\|\phi \circ \hat{d}^{j} g(y+\theta \lambda h)\right\| \leq N\|\phi\|\left\|d^{j} g(y+\theta \lambda h)\right\|,
$$

where $N=j^{j} / j$ ! .

From Lemmas 3.1 and 3.3, the map $d^{j} g: V \rightarrow \mathscr{P}\left({ }^{j} F, E^{* *}\right)$ is bounded on compact sets. So, it is locally bounded [8, Theorem 14.7]. Hence,

$$
\begin{aligned}
\lim _{t \rightarrow 0} & \frac{1}{|t|}\left\|d^{j-2} g(y+t h)-d^{j-2} g(y)-u_{j-1}^{y}(t h)\right\| \\
\quad & =\lim _{t \rightarrow 0} \sup _{\phi \in B_{E^{*}}} \frac{1}{|t|}\left\|\phi \circ\left(d^{j-2} g(y+t h)-d^{j-2} g(y)-u_{j-1}^{y}(t h)\right)\right\| \\
& \leq \lim _{t \rightarrow 0} C N\|\lambda h\|\|h\| \sup \left\{\left\|d^{j} g(y+\theta \lambda h)\right\|: \theta \in[0,1], \lambda \in[0, t]\right\}=0,
\end{aligned}
$$

uniformly for $h \in B$, and we conclude that $g: V \rightarrow E^{* *}$ is $(j-1)$-times differentiable. 
Finally, $d^{p-1} g$ is continuous. Indeed, let $K \subset V$ be a convex compact set, $x \in K$ fixed, $y \in K, h \in B_{F}$. By the mean value theorem

$$
\begin{aligned}
& \left\|d^{p-1} g(x)(h)-d^{p-1} g(y)(h)\right\| \\
& \quad=\sup \left\{\left|\phi \circ\left(d^{p-1} g(x)-d^{p-1} g(y)\right)(h)\right|: \phi \in B_{E^{*}}\right\} \\
& \quad \leq\|y-x\| \sup \left\{\left\|\phi \circ u_{p}^{z}(h)\right\|: z \in[x, y], \phi \in B_{E^{*}}\right\} \\
& \quad \leq\|y-x\| \sup \left\{\left\|\phi \circ \hat{d}^{p} g(z)\right\|: z \in[x, y], \phi \in B_{E^{*}}\right\} \\
& \quad \leq\|y-x\| L \sup \left\{\left\|d^{p} g(z)\right\|: z \in[x, y]\right\},
\end{aligned}
$$

where $L=p^{p} / p$ ! . Now, Lemma 3.3 allows us to conclude.

3.5. Remark. If $g \in C^{p}\left(V, E_{w^{*}}^{* *}\right)$, we cannot in general conclude that $g \in$ $C^{p}\left(V, E^{* *}\right)$. In $[16,11.3 .2]$ a function $g: \mathbb{R} \rightarrow c_{0}$ is given so that $g \notin$ $C^{1}\left(\mathbb{R}, l_{\infty}\right)$ and $\phi \circ g \in C^{1}(\mathbb{R})$ for each $\phi \in l_{1}$, so $g \in C^{1}\left(\mathbb{R},\left(l_{\infty}, w^{*}\right)\right)$.

3.6. Corollary. For $V \in \mathscr{O}(F)$, we have $C^{\infty}\left(V, E_{w^{*}}^{* *}\right)=C^{\infty}\left(V, E^{* *}\right)$.

Proof. By Proposition 3.4, $C^{\infty}\left(V, E_{w^{*}}^{* *}\right) \subset C^{\infty}\left(V, E^{* *}\right)$. The converse is clear.

We now show that a weakly $C^{p}$ mapping is of class $C^{p-1}$.

3.7. Corollary. Let $p \in \mathbb{N}^{*} \backslash\{0\}$ and $V \in \mathscr{O}(F)$. Suppose $g: V \rightarrow E$ is such that $\phi \circ g \in C^{p}(V)$ for each $\phi \in E^{*}$. Then $g \in C^{p-1}(V, E)$.

Proof. For $p=1$, the result follows from Theorem 3.2 and Proposition 3.4. For $p \geq 2$, applying 3.2, $g \in C^{p}\left(V, E_{w^{*}}^{* *}\right)$ and, from 3.4, $g \in C^{p-1}\left(V, E^{* *}\right)$. Now, given $y \in V, h \in F$ :

$$
d g(y)(h)=\lim _{t \rightarrow 0} t^{-1}[g(y+t h)-g(y)] .
$$

For every $t \neq 0, t^{-1}[g(y+t h)-g(y)] \in E$. Hence, the limit lies in $E$, and $g \in C^{1}(V, E)$. In the same way we can prove that $g \in C^{j}(V, E)$ whenever $1 \leq j \leq p-1$.

If $E_{w}$ denotes the space $E$ endowed with the weak topology, the following corollary is now immediate.

3.8. Corollary. Let $g: V \rightarrow E$ be a mapping, with $V \in \mathscr{O}(F)$. The following assertions are equivalent:

(a) $g \in C^{\infty}(V, E)$;

(b) for every $\phi \in E^{*}, \phi \circ g \in C^{\infty}(V)$;

(c) $g \in C^{\infty}\left(V, E_{w}\right)$.

The next example shows that given $p \in \mathbb{N} \backslash\{0\}$ and a mapping $g: V \rightarrow E$ such that $\phi \circ g \in C^{p}(V)$ for each $\phi \in E^{*}$, it is not in general true that $g \in$ $C^{p}\left(V, E_{w}\right)$, since the $p$ th derivative lies in $E^{* *}$.

3.9. Example. Let $g: \mathbb{R} \rightarrow c_{0}$ be the function given by $g(t)=\left(\psi_{n}(t)\right)_{n=1}^{\infty}$ where $\psi_{n}(t)=(1 / n) \sin n t \quad(t \in \mathbb{R})$. It is easy to check that $\phi \circ g \in C^{1}(\mathbb{R})$ for every $\phi=\left(\phi_{n}\right)_{n=1}^{\infty} \in l_{1}$, with

$$
(\phi \circ g)^{\prime}(t)=\sum_{n=1}^{\infty} \phi_{n} \psi_{n}^{\prime}(t)
$$


Hence, $g \in C^{1}\left(\mathbb{R},\left(l_{\infty}, w^{*}\right)\right)$. Furthermore,

$$
d g(0)=\left(\psi_{n}^{\prime}(0)\right)_{n=1}^{\infty}=(1,1,1, \ldots) \in l_{\infty} \backslash c_{0} .
$$

We remark that this map is weakly $C^{1}$ but is not differentiable.

\section{COMPOSITION OF DIFFERENTIABLE MAPPINGS}

The main result in this section states that if $V \in \mathscr{O}(F), U \in \mathscr{O}(E), g: V \rightarrow$ $U$ is a weakly $C^{p}$ mapping, and $f: U \rightarrow \mathbb{R}$ is of class $C^{p}$, then the composition map $f \circ g$ is of class $C^{p}$. Some stability properties of the classes $C_{c}^{p}(E, F)$ and $C_{w u}^{p}(E, F)$ under composition with $C^{p}$ functions are also analysed.

The proof of the following lemma is straightforward and omitted.

4.1. Lemma. If $X, Y_{1}, Y_{2}$ are locally convex spaces and $f_{i}: X \rightarrow Y_{i} \quad(i=$ $1,2)$ are differentiable mappings, then the map $f: X \rightarrow Y_{1} \times Y_{2}$ defined by $f(x)=\left(f_{1}(x), f_{2}(x)\right) \quad(x \in X)$ is differentiable and

$$
d f(x)(h)=\left(d f_{1}(x)(h), d f_{2}(x)(h)\right) \quad(x, h \in X) .
$$

4.2. Proposition. Let $p, m \in \mathbb{N}^{*} \backslash\{0\}, m \geq p$. If $U \in \mathscr{O}(E), V \in \mathscr{O}(F)$, and $g: V \rightarrow U$ is a mapping such that $\phi \circ g \in C^{p}(V)$ for every $\phi \in E^{*}$, then $f \circ g \in C^{p}(V)$ for each $f \in C^{m}(U)$.

Proof. By Theorem 3.2, $g \in C^{p}\left(V, E_{w^{*}}^{* *}\right)$.

(a) Let $p=1$. For $y \in V$, we have $d g(y) \in L\left(F, E_{w^{*}}^{* *}\right)$ and $d f(g(y)) \in E^{*}$. Following the argument of [22, 1.2.9], it is easy to see that $d(f \circ g)(y)=$ $d f(g(y)) \circ d g(y)$.

Moreover, $d(f \circ g): V \rightarrow F^{*}$ is continuous. Indeed, let $\left(y_{n}\right) \subset V$ be a sequence converging to $y \in V$. Since $d g: V \rightarrow \mathscr{P}\left({ }^{1} F, E_{w^{*}}^{* *}\right)$ is continuous, for $\varepsilon>0$, there is $n_{1} \in \mathbb{N}$ so that $\left(d g\left(y_{n}\right)-d g(y)\right)(z) \in(\varepsilon / 2)\{d f(g(y))\}^{\circ}$ $\left(z \in B_{F}\right)$ for $n \geq n_{1}$. Let $M=\sup \left\{\left\|d g\left(y_{n}\right)\right\|: n \in \mathbb{N}\right\}<\infty$ (Lemma 3.3). There is $\eta>0$ such that whenever $\|g(y)-x\|<\eta$, for $x \in U$, we have $\|d f(g(y))-d f(x)\|<\varepsilon / 2 M$. Since $g$ is continuous (Corollary 3.7), there is $n_{2} \in \mathbb{N}$ so that $\left\|g\left(y_{n}\right)-g(y)\right\|<\eta$ for $n \geq n_{2}$.

Therefore, for $n \geq \max \left\{n_{1}, n_{2}\right\}$ we have

$$
\begin{aligned}
& \left\|d f\left(g\left(y_{n}\right)\right) \circ d g\left(y_{n}\right)-d f(g(y)) \circ d g(y)\right\| \\
& \quad \leq\left\|d f\left(g\left(y_{n}\right)\right) \circ d g\left(y_{n}\right)-d f(g(y)) \circ d g\left(y_{n}\right)\right\| \\
& \quad+\left\|d f(g(y)) \circ d g\left(y_{n}\right)-d f(g(y)) \circ d g(y)\right\| \\
& \quad<\varepsilon .
\end{aligned}
$$

(b) For arbitrary $p$, the proof needs too complicated notations. Therefore, we only prove the result for the case $p=2$, which contains all the ideas needed in the general case. By $(\mathrm{a}), d(f \circ g)$ is the composition of the following two maps:

$$
\begin{gathered}
S: y \in V \mapsto(d g(y), d f(g(y))) \in L\left(F, E_{w^{*}}^{* *}\right) \times E^{*}, \\
T:(A, \phi) \in L\left(F, E_{w^{*}}^{* *}\right) \times E^{*} \mapsto \phi \circ A \in F^{*} .
\end{gathered}
$$

$T$ is differentiable $[22,3.1 .1]$, and so is $S$ by the preceding lemma. Thus, applying the chain rule $[22,1.2 .9]$ :

$$
\begin{aligned}
d(T \circ S)(y)(h) & =d T(S(y))(d S(y)(h)) \\
& =d f(g(y)) \circ d(d g)(y)(h)+d(d f)(g(y))(d g(y)(h)) \circ d g(y),
\end{aligned}
$$


where we have applied the formula giving the derivative of the composition map [22, 3.1.1]. Hence,

$$
\begin{aligned}
\hat{d}^{2}(f \circ g)(y)\left(h_{1}, h_{2}\right)= & d f(g(y))\left(\hat{d}^{2} g(y)\left(h_{1}, h_{2}\right)\right) \\
& +\hat{d}^{2} f(g(y))\left(d g(y)\left(h_{1}\right), d g(y)\left(h_{2}\right)\right) .
\end{aligned}
$$

(c) Finally, $d^{2}(f \circ g): V \rightarrow \mathscr{P}\left({ }^{2} F\right)$ is continuous. Indeed, let $\left(y_{n}\right) \subset V$ be a sequence converging to $y \in V$. By Lemmas 3.1 and 3.3, we can take

$$
M=\max \left\{1, \sup \left\{\left\|\hat{d}^{2} f\left(g\left(y_{n}\right)\right)\right\|,\left\|d g\left(y_{n}\right)\right\|^{2},\left\|d^{2} g\left(y_{n}\right)\right\|: n \in \mathbb{N}\right\}\right\}<\infty .
$$

Given $\varepsilon>0$, there is $n_{0} \in \mathbb{N}$ so that for $n \geq n_{0}$ we have

$\left\|d g\left(y_{n}\right)-d g(y)\right\|<\varepsilon / 8 M^{2}$,

$\left\|d^{2} f\left(g\left(y_{n}\right)\right)-d^{2} f(g(y))\right\|<\varepsilon / 4 M$,

$\left\|d f\left(g\left(y_{n}\right)\right)-d f(g(y))\right\|<\varepsilon / 4 M$,

$\left|d f(g(y))\left(d^{2} g\left(y_{n}\right)(h)-d^{2} g(y)(h)\right)\right|<\varepsilon / 4\left(h \in B_{F}\right)$,

since $\left(d^{2} g\left(y_{n}\right)-d^{2} g(y)\right)_{n=1}^{\infty}$ converges to zero in $\mathscr{P}\left({ }^{2} F, E_{w^{*}}^{* *}\right)$. A routine calculation now shows that, for $n \geq n_{0}$,

$$
\left|d^{2}(f \circ g)\left(y_{n}\right)(h)-d^{2}(f \circ g)(y)(h)\right|<\varepsilon \quad\left(h \in B_{F}\right) .
$$

4.3. Proposition. Let $p \in \mathbb{N}^{*} \backslash\{0\}, W \in \mathscr{O}(G), V \in \mathscr{O}(F), g \in C_{c}^{p}(W, V)$, and $f \in C^{p}(V, E)$. Then $f \circ g \in C_{c}^{p}(W, E)$.

Proof. The derivatives $d^{j} g(x)(x \in W, 1 \leq j \leq p)$ are weakly continuous on bounded subsets of $G$. Taking the chain rule into account $[22,1.8 .3]$, it is easy to check that the polynomial $d^{j}(f \circ g)(x)$ is weakly continuous on bounded subsets, and so $f \circ g \in C_{c}^{p}(W, E)$.

In the next proposition we use the fact that for $A \in L\left({ }^{k} F, E\right)$, we have

$$
\begin{aligned}
&\left\|A\left(u_{1}, u_{2}, \ldots, u_{k}\right)-A\left(v_{1}, v_{2}, \ldots, v_{k}\right)\right\| \\
& \leq\left\|A\left(u_{1}, u_{2}, \ldots, u_{k}\right)-A\left(u_{1}, u_{2}, \ldots, u_{k-1}, v_{k}\right)\right\| \\
&+\left\|A\left(u_{1}, u_{2}, \ldots, u_{k-1}, v_{k}\right)-A\left(u_{1}, u_{2}, \ldots, v_{k-1}, v_{k}\right)\right\| \\
&+\cdots+\left\|A\left(u_{1}, v_{2}, \ldots, v_{k}\right)-A\left(v_{1}, v_{2}, \ldots, v_{k}\right)\right\| \\
& \leq\|A\| \sum_{r=1}^{k}\left\|u_{1}\right\| \cdots\left\|u_{r-1}\right\|\left\|u_{r}-v_{r}\right\|\left\|v_{r+1}\right\| \cdots\left\|v_{k}\right\| .
\end{aligned}
$$

4.4. Proposition. Let $p \in \mathbb{N}^{*} \backslash\{0\}, W \in \mathscr{O}(G), V \in \mathscr{O}(F), g \in C_{w u}^{p}(W, V)$, and $f \in C^{p}(V, E)$. Then $f \circ g \in C_{w u}^{p}(W, E)$.

Proof. It is enough to show that, for $0 \leq j \leq p$, the map $d^{j}(f \circ g): W \rightarrow$ $\mathscr{P}_{w b}\left({ }^{j} G, E\right)$ is weakly uniformly continuous on bounded sets. This is clear for $j=0$. For $1 \leq j \leq p$ ( $p$ finite), let $B \subseteq W$ be bounded, $x \in W$, and $y \in G$. By the chain rule $[22,1.8 .3], d^{j}(f \circ g)(x)(y)$ is a finite sum of terms of the form

$$
\hat{d}^{k} f(g(x))\left(d^{i_{1}} g(x)(y), \ldots, d^{i_{k}} g(x)(y)\right)
$$

where $1 \leq k \leq j$ and $i_{1}+\cdots+i_{k}=j$. Since $d^{i_{r}} g(B) \subset \mathscr{P}\left({ }^{i_{r}} G, F\right)$ and $\hat{d}^{k} f(g(B)) \subset L\left({ }^{k} F, E\right)$ are precompact (see $\left.\S 1\right)$, we can take

$$
M=\max \left\{1, \sup \left\{\left\|\hat{d}^{k} f(g(x))\right\|,\left\|d^{i_{r}} g(x)\right\|: x \in B, 1 \leq r \leq k\right\}\right\}<\infty .
$$


Given $\varepsilon>0$, there is $\eta>0$ such that whenever $\left\|g\left(x_{1}\right)-g\left(x_{2}\right)\right\|<\eta\left(x_{1}, x_{2} \in\right.$ $B)$, we have

$$
\left\|\hat{d}^{k} f\left(g\left(x_{1}\right)\right)-\hat{d}^{k} f\left(g\left(x_{2}\right)\right)\right\|<\varepsilon / 2 M^{j} .
$$

Moreover, there is a weak zero neighborhood $W$ in $G$, such that, whenever $x_{1}-x_{2} \in W \quad\left(x_{1}, x_{2} \in B\right)$, we have

$\left\|g\left(x_{1}\right)-g\left(x_{2}\right)\right\|<\eta \quad$ and $\quad\left\|d^{i_{r}} g\left(x_{1}\right)-d^{i_{r}} g\left(x_{2}\right)\right\|<\varepsilon / 2 j M^{j} \quad(1 \leq r \leq k)$.

To simplify notation, we write

$$
Q(u, v)=\hat{d}^{k} f(g(u))\left(d^{i_{1}} g(v)(y), \ldots, d^{i_{k}} g(v)(y)\right) \text {. }
$$

For $y \in B_{G}$, and $x_{1}, x_{2} \in B$ with $x_{1}-x_{2} \in W$, we have

$$
\begin{aligned}
\left\|Q\left(x_{1}, x_{1}\right)-Q\left(x_{2}, x_{2}\right)\right\| & \leq\left\|Q\left(x_{1}, x_{1}\right)-Q\left(x_{1}, x_{2}\right)\right\|+\left\|Q\left(x_{1}, x_{2}\right)-Q\left(x_{2}, x_{2}\right)\right\| \\
\leq & \left\|\hat{d}^{k} f\left(g\left(x_{1}\right)\right)\right\| \sum_{r=1}^{k}\left\|d^{i_{1}} g\left(x_{1}\right)(y)\right\| \cdots\left\|d^{i_{r-1}} g\left(x_{1}\right)(y)\right\| \\
& \cdot\left\|d^{i_{r}} g\left(x_{1}\right)(y)-d^{i_{r}} g\left(x_{2}\right)(y)\right\|\left\|d^{i_{r+1}} g\left(x_{2}\right)(y)\right\| \cdots\left\|d^{i_{k}} g\left(x_{2}\right)(y)\right\| \\
& +\left\|\hat{d}^{k} f\left(g\left(x_{1}\right)\right)-\hat{d}^{k} f\left(g\left(x_{2}\right)\right)\right\| \prod_{r=1}^{k}\left\|d^{i_{r}} g\left(x_{2}\right)(y)\right\| \\
< & k M^{k} \frac{\varepsilon}{2 j M^{j}}+\frac{\varepsilon}{2 M^{j}} M^{k} \leq \varepsilon .
\end{aligned}
$$

Hence, $f \circ g \in C_{w u}^{P}(W, E)$. Slight modifications are needed for $p=\infty$.

\section{ChaRACTERIZATIONS OF HOMOMORPHISMS BETWEeN ALgEBRAS OF DIFFERENTIABLE FUNCTIONS}

We prove that the continuous homomorphisms between algebras of Hadamard differentiable functions are exactly those induced by Hadamard differentiable mappings. Later on, we give one of the main results: namely, for $m \geq p$, the continuous homomorphisms from $C^{m}(U)$ into $C^{p}(V)$ when both algebras are endowed with the compact open topology, are exactly those induced by the weakly $C^{p}$ mappings from $V$ to $U$. The continuous homomorphisms for the compact-compact topology are also described. Finally, we analyse the relationship among continuities for different topologies and we conclude with a brief note on automatic continuity.

Let us recall that, for a Hausdorff locally convex space $X$, the $\varepsilon$-product $X \varepsilon E=L_{\varepsilon}\left(E_{c}^{*}, X\right)$ is the space of all continuous linear operators from $E_{c}^{*}$ to $X$ endowed with the topology of uniform convergence on equicontinuous subsets of $E^{*}$. The following result will be needed:

5.1. Proposition. For $V \in \mathscr{O}(F)$ and $m \in \mathbb{N}^{*} \backslash\{0\}$, the following topological isomorphisms hold:

(a) $C_{H}^{m}(V, E) \simeq C_{H}^{m}(V) \varepsilon E[6$, Theorem 10];

(b) $\left(C_{w u}^{m}(V, E), \tau_{b}^{m}\right) \simeq\left(C_{w u}^{m}(V), \tau_{b}^{m}\right) \varepsilon E$ [16, Theorem 8.1.1];

(c) $\left(C_{K}^{m}(V, E), \tau_{u}^{m}\right) \simeq\left(C^{m}(V), \tau_{u}^{m}\right) \varepsilon E[1$, p. 220];

(d) $\left(C_{c}^{m}(V, E), \tau_{u}^{m}\right) \simeq\left(C_{c}^{m}(V), \tau_{u}^{m}\right) \varepsilon E$ [16, Theorem 8.1.4]. 
All these isomorphisms are defined in the following way: to each $f$ in the first space, we associate the operator $\phi \mapsto \phi \circ f$ in the E-product.

In order to characterize the homomorphisms between algebras of Hadamard differentiable functions, we need a preliminary lemma:

5.2. Lemma. Let $p \in \mathbb{N}^{*} \backslash\{0\}, V \in \mathscr{O}(F)$, and $g \in C_{H}^{p}(V, E)$. If $K \subset V$ is a compact set, then $d^{j} g(K)(K)$ is compact in $E$, for $1 \leq j \leq p$.

Proof. It is enough to prove that the map $\Phi: K \times K \rightarrow E$ given by $\Phi(x, y)=$ $d^{j} g(x)(y)$ is continuous. Let $\left(\left(x_{n}, y_{n}\right)\right)_{n=1}^{\infty}$ be a sequence in $K \times K$ converging to $(x, y)$ and let $\varepsilon>0$. Using the continuity of $d^{j} g: V \rightarrow \mathscr{P}\left({ }^{j} F, E\right)_{c}$, we can find $n_{0} \in \mathbb{N}$ so that for $n \geq n_{0}$ we have

$$
\left\|\left(d^{j} g\left(x_{n}\right)-d^{j} g(x)\right)(h)\right\|<\varepsilon / 2 \quad(h \in K)
$$

and

Therefore,

$$
\left\|d^{j} g(x)\left(y_{n}\right)-d^{j} g(x)(y)\right\|<\varepsilon / 2 .
$$

$$
\left\|d^{j} g\left(x_{n}\right)\left(y_{n}\right)-d^{j} g(x)(y)\right\|<\varepsilon .
$$

5.3. Theorem. Let $p, m \in \mathbb{N}^{*} \backslash\{0\}, m \geq p, U \in \mathscr{O}(E), V \in \mathscr{O}(F)$. The nonzero continuous homomorphisms $A: C_{H}^{m}(U) \rightarrow C_{H}^{p}(V)$ are exactly those induced by the mappings $g \in C_{H}^{p}(V, U)$ by means of the formula $A(f)=f \circ g$ $\left(f \in C_{H}^{m}(U)\right)$.

Proof. Let $A$ be a nonzero continuous homomorphism. By the comment after Theorem 2.2, there is a mapping $g: V \rightarrow U$ such that $A(f)=f \circ g(f \in$ $\left.C_{H}^{m}(U)\right)$. Moreover, $\left.A\right|_{E^{*}}: E_{c}^{*} \rightarrow C_{H}^{p}(V)$ belongs to $C_{H}^{p}(V) \varepsilon E$. By Proposition 5.1(a), there is a mapping $g_{1} \in C_{H}^{p}(V, U)$ such that $\left.A\right|_{E^{*}}(\phi)=\phi \circ g_{1} \quad\left(\phi \in E^{*}\right)$. Thus, $\phi \circ g=A(\phi)=\phi \circ g_{1} \quad\left(\phi \in E^{*}\right)$, so for each $y \in V, \phi(g(y))=\phi\left(g_{1}(y)\right)$ $\left(\phi \in E^{*}\right)$, and hence $g=g_{1}$. Conversely, given $g \in C_{H}^{p}(V, U)$, we define the homomorphism $A(f)=f \circ g\left(f \in C_{H}^{m}(U)\right)$. Let $\left(f_{\alpha}\right)$ be a net converging to zero in $C_{H}^{m}(U)$. If $K \subset V$ is compact then, applying Lemma 5.2 and the chain rule $[22,1.8 .3]$, we see that $\left(f_{\alpha} \circ g\right)$ converges to zero in $C_{H}^{p}(V)$, so $A$ is continuous.

Another lemma, analogous to 3.3 and 5.2, is needed:

5.4. Lemma. Let $p \in \mathbb{N}^{*} \backslash\{0\}, V \in \mathscr{O}(F)$, and $j \in\{1, \ldots, p\}$. Then:

(a) for $g \in C_{w u}^{p}(V, E)$ and $B \subseteq V$ bounded, $d^{j} g(B)\left(B_{F}\right) \subset E$ is precompact;

(b) for $g \in C_{K}^{p}(V, E)$ and $K \subset V$ compact, $d^{j} g(K)\left(B_{F}\right) \subset E$ is precompact.

Proof. (a) Since $d^{j} g(B) \subset \mathscr{P}_{w b}\left({ }^{j} F, E\right)$ is precompact (see $\S 1$ ), given $\varepsilon>0$, we can find $x_{1}, \ldots, x_{n} \in B$ such that for every $x \in B$ there is $k \in\{1, \ldots, n\}$ for which $\left\|d^{j} g(x)-d^{j} g\left(x_{k}\right)\right\|<\varepsilon / 2$. By the precompactness of $d^{j} g\left(x_{k}\right)\left(B_{F}\right)$, we can find a finite subset $S_{k} \subset B_{F}$ such that, for any $y \in B_{F}$, there is $z \in S_{k}$ with $\left\|d^{j} g\left(x_{k}\right)(y)-d^{j} g\left(x_{k}\right)(z)\right\|<\varepsilon / 2$. Then, for $x \in B$ and $y \in B_{F}$, there exist $k \in\{1, \ldots, n\}$, a finite subset $S_{k} \subset B_{F}$ and $z \in S_{k}$ such that

$$
\begin{aligned}
\left\|d^{j} g(x)(y)-d^{j} g\left(x_{k}\right)(z)\right\| \leq & \left\|d^{j} g(x)(y)-d^{j} g\left(x_{k}\right)(y)\right\| \\
& +\left\|d^{j} g\left(x_{k}\right)(y)-d^{j} g\left(x_{k}\right)(z)\right\| \\
& <\varepsilon . \quad \square
\end{aligned}
$$


(b) Since $d^{j} g(K)$ is compact in $\mathscr{P}_{K}\left({ }^{j} F, E\right)$ and, for $x \in V, d^{j} g(x)\left(B_{F}\right)$ is precompact in $E$, the proof is like in (a).

One of the main purposes of this study was the description of the continuous homomorphisms for the compact open topology, which is contained in (a) of the next theorem. The other assertions show that when the domain algebra is endowed with the compact-compact topology, then the inducing mapping is continuously differentiable up to the order of the range algebra.

5.5. Theorem. Let $p, m \in \mathbb{N}^{*} \backslash\{0\}, m \geq p, U \in \mathscr{O}(E), V \in \mathscr{O}(F)$. The nonzero continuous homomorphisms $A$ in the table are exactly those induced by the corresponding mappings $g: V \rightarrow U$, by means of the formula $A(f)=f \circ g$ for every $f \in C^{m}(U)$ :

\begin{tabular}{c|c}
\hline Homomorphism $A$ & Inducing map $g: V \rightarrow U$ \\
\hline$\left(\right.$ a) $\left(C^{m}(U), \tau_{u}^{m}\right) \rightarrow\left(C^{p}(V), \tau_{u}^{p}\right)$ & $\phi \circ g \in C^{p}(V)$ for each $\phi \in E^{*}$ \\
\hline (b) $\left(C^{m}(U), \tau_{c}^{m}\right) \rightarrow\left(C^{p}(V), \tau_{c}^{p}\right)$ & $g \in C_{H}^{p}(V, U)$ and \\
& $\phi \circ g \in C^{p}(V)$ for each $\phi \in E^{*}$ \\
\hline (c) $\left(C^{m}(U), \tau_{c}^{m}\right) \rightarrow\left(C_{w u}^{p}(V), \tau_{b}^{p}\right)$ & $g \in C_{w u}^{p}(V, U)$ \\
\hline (d) $\left(C^{m}(U), \tau_{c}^{m}\right) \rightarrow\left(C^{p}(V), \tau_{u}^{p}\right)$ & $g \in C_{K}^{p}(V, U)$ \\
\hline (e) $\left(C^{m}(U), \tau_{c}^{m}\right) \rightarrow\left(C_{c}^{p}(V), \tau_{u}^{p}\right)$ & $g \in C_{c}^{p}(V, U)$ \\
\hline
\end{tabular}

Proof. The proof is similar to that of Theorem 5.3. We only quote the previous results needed in each case:

(a) uses Proposition 4.2, Lemma 3.3, and Theorem 2.2.

(b) uses Proposition 5.1(a) and Theorem 5.3, since $\left(C^{m}(U), \tau_{c}^{m}\right)$ is a subspace of $C_{H}^{m}(U)$, and Proposition 4.2.

(c) uses Propositions 5.1(b) and 4.4 and Lemma 5.4(a).

(d) uses Proposition 5.1(c) and Lemma 5.4(b).

(e) needs Propositions 5.1(d) and 4.3 and Lemma 5.4(b), since $C_{c}^{p}(V, U) \subseteq$ $C_{K}^{p}(V, U)$.

5.6. Remarks. (a) The nondifferentiable map $g: \mathbb{R} \rightarrow c_{0}$ of Example 3.9 induces a continuous homomorphism from $\left(C^{1}\left(c_{0}\right), \tau_{u}^{1}\right)$ to $C^{1}(\mathbb{R})$.

(b) Not every mapping $g \in C_{H}^{p}(V, U)$ satisfies $\phi \circ g \in C^{p}(V) \quad\left(\phi \in E^{*}\right)$. Indeed, if $E=\mathbb{R}$ and $F=L^{1}[0,1]$, in [21, 2.2] an example is given of a mapping $g \in C_{H}^{1}(F, E)$ which is not (weakly) differentiable.

(b) Neither can we drop condition $g \in C_{H}^{p}(V, U)$ in Theorem 5.5(b). Indeed, the function $g: \mathbb{R} \rightarrow c_{0}$ of 3.5 satisfies $\phi \circ g \in C^{1}\left(c_{0}\right) \quad\left(\phi \in l_{1}\right)$, but does not belong to $C_{H}^{1}\left(\mathbb{R}, c_{0}\right)=C^{1}\left(\mathbb{R}, c_{0}\right)$.

In the following corollary, for $U \in \mathscr{O}(E)$ and $V \in \mathscr{O}(F)$, we say that a homomorphism $A: C^{m}(U) \rightarrow C^{p}(V)$ is $\tau_{1}-\tau_{2}$ continuous if it is continuous when $C^{m}(U)$ and $C^{p}(V)$ are endowed with the topologies $\tau_{1}$ and $\tau_{2}$ respectively.

5.7. Corollary. Let $A: C^{m}(U) \rightarrow C^{p}(V)$ be an algebra homomorphism, with $p, m \in \mathbb{N}^{*} \backslash\{0\}, m \geq p$. We consider the following assertions:

(a) $A$ is $\tau_{u}^{m}-\tau_{u}^{p}$ continuous; 
(b) $A$ is $\tau_{u}^{m}-\tau_{c}^{p}$ continuous;

(c) $A$ is $\tau_{c}^{m}-\tau_{c}^{p}$ continuous.

Then $(\mathrm{a}) \Leftrightarrow(\mathrm{b}) \Leftarrow(\mathrm{c})$. For $p=\infty$, all three are equivalent.

Proof. (c) $\Rightarrow$ (b) and (a) $\Rightarrow$ (b). Obvious.

(b) $\Rightarrow$ (a). Apply Theorems 2.2 and 5.5(a).

In general, (a) does not imply (c): the mapping $g: \mathbb{R} \rightarrow c_{0}$ of Remark 5.6(c) induces a $\tau_{u}^{1}-\tau_{u}^{1}$ continuous homomorphism from $C^{1}\left(c_{0}\right)$ into $C^{1}(\mathbb{R})$ which is not $\tau_{c}^{1}-\tau_{c}^{1}$ continuous. The equivalence of the three assertions for $p=\infty$ follows from the fact that $C_{H}^{\infty}(V, U)$ and $C^{\infty}(V, U)$ coincide as linear spaces [9, Proposition 2.6 and Remark].

Up to now, we have excluded the possibility $m<p$. The reason is that, for $m<p$, any continuous homomorphism $A: C^{m}(U) \rightarrow C^{p}(V)$, when $C^{m}(U)$ is endowed with one of the topologies $\tau_{u}^{m}$ or $\tau_{c}^{m}$ and $C^{p}(V)$ is endowed with $\tau_{u}^{p}$ or $\tau_{c}^{p}$, is induced by a constant mapping $g: V \rightarrow U$. This can be proved by a slight modification of $[16,11.2 .7]$.

We end with a short analysis on automatic continuity. We first state a result on real-valued homomorphisms, due to Jaramillo.

5.8. Theorem [12]. Let $m \in \mathbb{N}^{*} \backslash\{0\}$ and $U \in \mathscr{O}(E)$, where $E$ satisfies one of the following conditions:

(a) $E^{*}$ is $w^{*}$-separable;

(b) $E$ has nonmeasurable cardinal and admits $C^{m}$-partitions of unity;

(c) $E$ is a closed subspace of $C(K)$ where $K$ is a separable compact space;

(d) $E$ is a closed subspace of a superreflexive space with nonmeasurable cardinal.

Then, any nonzero homomorphism $\Phi: C^{m}(U) \rightarrow \mathbb{R}$ is a point evaluation in the sense that there is a unique $x \in U$ such that $\Phi(f)=f(x)$ for each $f \in C^{m}(U)$.

Condition (a) is fulfilled by separable spaces and their duals. Condition (b) is satisfied, for instance, by $c_{0}(I)$ where $I$ is any index set and $L^{2 n}(\mu)$ for $n \in \mathbb{N}$ and any measure $\mu$. The definition of measurable cardinal may be seen in [5, 1.7]. Anyway, the condition imposed in (b) and (d) to the cardinal of $E$ is very mild because it is not known whether any measurable cardinal exists.

5.9. Corollary. Let $p, m \in \mathbb{N}^{*} \backslash\{0\}, U \in \mathscr{O}(E)$, and $V \in \mathscr{O}(F)$, where $E$ satisfies any of the conditions of the previous theorem. Then every homomorphism $A: C^{m}(E) \rightarrow C^{p}(F)$ is automatically continuous when both algebras are endowed with the compact open topology of order $m$ and $p$ respectively.

Proof. The argument of Theorem 2.2 provides a mapping $g: V \rightarrow U$ such that $A(f)=f \circ g\left(f \in C^{m}(U)\right)$. Since $\phi \circ g \in C^{p}(V)$ for any $\phi \in E^{*}$, we conclude from Theorem 5.5(a) that $A$ is $\tau_{u}^{m}-\tau_{u}^{p}$ continuous.

It is interesting to remark that however there are discontinuous homomorphisms from $\left(C^{1}\left(c_{0}\right), \tau_{c}^{1}\right)$ to $C^{1}(\mathbb{R})$ (see Corollary 5.7).

The authors are grateful to the referee for his very valuable suggestions.

Added in proof. In a paper to appear in Monatsh. Math., Biström and Lindström have proved that Theorem 5.8 is valid for a larger class of Banach spaces, including the $W \subset G$ spaces and their duals. 


\section{REFERENCES}

1. R. M. Aron, Compact polynomials and compact differentiable mappings between Banach spaces, Séminaire P. Lelong (Analyse), Lecture Notes in Math., vol. 524, Springer-Verlag, Berlin, 1976, pp. 213-222.

2. R. M. Aron, J. Gómez, and J. G. Llavona, Homomorphisms between algebras of differentiable functions in infinite dimensions, Michigan Math. J. 35 (1988), 163-178.

3. R. M. Aron and J. G. Llavona, Composition of weakly uniformly continuous functions, Proc. Roy. Irish Acad. Sect. A 88(1) (1988), 29-33.

4. R. M. Aron and J. B. Prolla, Polynomial approximation of differentiable functions on Banach spaces, J. Reine Angew. Math. 313 (1980), 195-216.

5. E. Beckenstein, L. Narici, and C. Suffel, Topological algebras, Math. Stud., vol. 24, NorthHolland, Amsterdam, 1977.

6. F. Bombal and J. G. Llavona, La propiedad de aproximación en espacios de funciones diferenciables, Rev. Real Acad. Cienc. Exact. Fís. Natur. Madrid 70(4) (1976), 727-741.

7. N. Bourbaki, Éléments de mathématique. Fasc. XXXIII. Variétés différentielles et analytiques. Fascicule de résultats, Actualités Sci. Indust., no. 1333, Hermann, Paris, 1971.

8. S. B. Chae, Holomorphy and calculus in normed spaces, Monographs Textbooks Pure Appl. Math., vol. 92, Dekker, New York, 1985.

9. J. F. Colombeau and R. Meise, $C^{\infty}$-functions on locally convex and on bornological vector spaces, Functional Analysis, Holomorphy and Approximation Theory, Lecture Notes in Math., vol. 843, Springer-Verlag, Berlin, 1981, pp. 195-216.

10. J. Gómez, Espectro e ideales primarios del álgebra $C_{w b}^{p}(E)$ de funciones débilmente diferenciables sobre un espacio de Banach, Rev. Real Acad. Cienc. Exact. Fis. Natur. Madrid 75(2) (1981), pp. 514-519.

11. J. A. Jaramillo, An example on composite differentiable functions in infinite dimensions, Bull. Austral. Math. Soc. 40(1) (1989), 91-95.

12. $\ldots$, Multiplicative functionals on algebras of differentiable functions, Arch. Math. 58 (1992), 384-387.

13. J. A. Jaramillo and J. G. Llavona, Homomorphisms between algebras of continuous functions, Canad. J. Math. 41(1) (1989), 132-162.

14. H. H. Keller, Differential calculus in locally convex spaces, Lecture Notes in Math., vol. 417, Springer-Verlag, Berlin, 1974.

15. J. G. Llavona, Approximations of differentiable functions, Adv. Math. Suppl. Stud. 4(1979), 197-221.

16. __ Approximation of continuously differentiable functions, Math. Stud., vol. 130, NorthHolland, Amsterdam, 1986.

17. J. Mujica, Complex analysis in Banach spaces, Math. Stud., vol. 120, North-Holland, Amsterdam, 1986.

18. L. Nachbin, Topology on spaces of holomorphic mappings, Ergeb. Math. Grenzgeb., vol. 47, Springer-Verlag, Berlin, 1969.

19. J. B. Prolla, On polynomial algebras of continuously differentiable functions, Atti Accad. Naz. Lincei Rend. Cl. Sci. Fis. Mat. Natur. (8) 57 (1974), 481-486.

20. L. Schwartz, Espaces de fonctions différentiables à valeurs vectorielles, J. Analyse Math. 4 (1954), 88-148.

21. M. Sova, Conditions of differentiability in linear topological spaces, Czechoslovak Math. J. 16(91) (1966), 339-362. (Russian)

22. S. Yamamuro, Differential calculus in topological linear spaces, Lecture Notes in Math., vol. 374, Springer-Verlag, Berlin, 1974.

Departamento de Matemática Aplicada, ETS de Ingenieros Industriales, Universidad Politécnica de Madrid, C. José Gutiérrez Abascal 2, 28006 Madrid, Spain

Departamento de análisis Matemático, Facultad de Ciencias Matemáticas, Universidad Complutense de Madrid, 28040 Madrid, SPain 